\title{
Incorporating simulation into gynecologic surgical training
}

\author{
Kyle Wohlrab, MD; J. Eric Jelovsek, MD, MMEd; Deborah Myers, MD
}

\section{Introduction}

While there are more surgical options for women, there has been a decrease in overall gynecologic surgery over the past 30 years. ${ }^{1}$ This issue, combined with changes in residency education (including restrictions on work hours), has led to a dilemma in the surgical teaching of obstetrics and gynecology residents that forces educators to ask the questions "Which surgical procedures should every Ob-Gyn resident know how to perform, and how do we ensure that graduating residents have achieved competency in those procedures?" Unfortunately, we have not been able to answer those questions; a survey of graduating obstetrics and gynecology residents reported that many feel surgically underprepared. ${ }^{2}$ Fortunately, simulation training is available to complement surgical education and to address the complex needs of training programs. This article will review the important learning concepts and components of a simulation-based educational curriculum, and provide a framework for its implementation.

\section{Learning through simulation}

Although Dr Halsted was instrumental in bringing resident education out of the classroom and into the operating room,

From Warren Alpert Medical School of Brown University, Division of Female Pelvic Medicine and Reconstructive Surgery, Women \& Infants Hospital, Providence, RI (Drs Wohlrab and Myers), and Obstetrics, Gynecology, and Women's Health Institute and Simulation and Advanced Skills Center, Cleveland Clinic, Cleveland, $\mathrm{OH}$ (Dr Jelovsek).

Received Sept. 20, 2016; revised March 22 , 2017; accepted May 7, 2017.

The authors report no conflict of interest.

Corresponding author: Kyle Wohlrab, MD. kwohlrab@wihri.org

0002-9378/\$36.00

(c) 2017 Elsevier Inc. All rights reserved. http://dx.doi.org/10.1016/j.ajog.2017.05.017

Today's educational environment has made it more difficult to rely on the Halstedian model of "see one, do one, teach one" in gynecologic surgical training. There is decreased surgical volume, but an increased number of surgical modalities. Fortunately, surgical simulation has evolved to fill the educational void. Whether it is through skill generalization or skill transfer, surgical simulation has shifted learning from the operating room back to the classroom. This article explores the principles of surgical education and ways to introduce simulation as an adjunct to residency training. We review high- and low-fidelity surgical simulators, discuss the progression of surgical skills, and provide options for skills competency assessment. Time and money are major hurdles when designing a simulation curriculum, but low-fidelity models, intradepartmental cost sharing, and utilizing local experts for simulation proctoring can aid in developing a simulation program.

Key words: gynecologic surgery, simulation training, skill generalization, skill transfer, surgical repetition, surgical training

the current trend of decreasing surgical volume requires a shift in learning back to the classroom. ${ }^{3}$ Furthermore, rapid surgical innovation in minimally invasive procedures, devices, and surgical techniques have complicated the learning landscape. Gynecologic surgeons are inundated with new products, so we must provide our residents with a solid knowledge and surgical foundation to evaluate new techniques and technology, similar to critiquing a journal article. Surgical simulation allows trainees to practice and develop surgical skills, troubleshoot and try equipment, and compare competing devices. Resident training should prepare new physicians to offer the safest, most efficient, and most cost-effective surgery available.

\section{Learning concepts \\ Skill learning}

Gallagher and colleagues ${ }^{4}$ describe skills acquisition through both skill generalization and skill transfer. Skill generalization, such as moving inanimate objects around a laparoscopic field, is the practice of a basic skill needed for an operation. Skill transfer, such as intracorporeal knot-tying on a segment of large bowel, involves the practice of a particular procedure replicated in a simulated environment. Another example of a skill transfer model would be residents practicing laparoscopic hysterectomy skills on a cadaver or animal model. Often skill transfer exercises are costly and time-consuming to develop. Skill generalization is easier to implement into educational curricula since the trainee does not have to worry about lack of realism. In fact, many highfidelity laparoscopic and robotic trainers now include software packages with games that involve skill generalization that bridges the gap between simulation and surgical experience. Combining distinct skills needed to perform an operation is analogous to an athlete or dancer who practices a difficult move over and over only to combine it into a host of other steps for a performance. The Fundamentals of Laparoscopic Surgery (FLS) is a competency-based exam required by the American Board of Surgery that employs a skill generalization model. The exam includes core skills of laparoscopy such as cutting a circle from gauze, a peg transfer exercise, and use of an endoscopic loop. FLS has gained widespread acceptance and completion is a requirement at some 


\section{FIGURE 1 \\ Low-fidelity laparoscopic simulation}

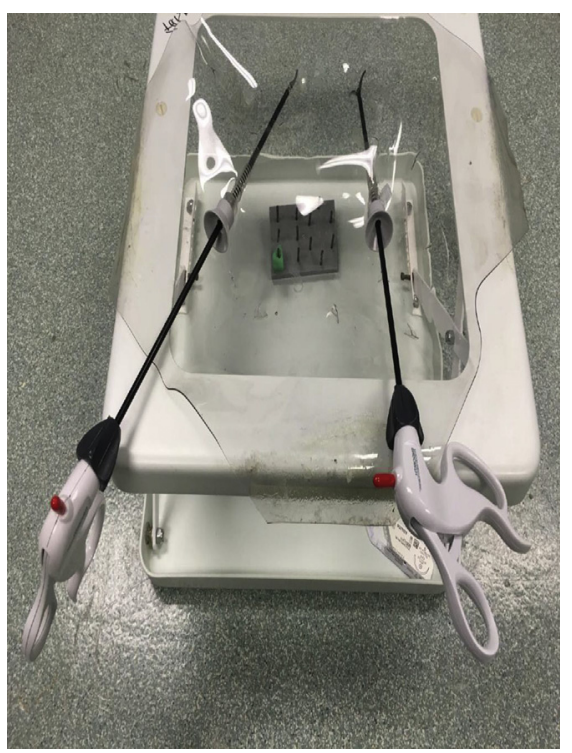

Laparoscopic box trainer with peg transfer exercise and 2 laparoscopic instruments.

Wohlrab. Simulation and gynecologic surgical training. Am J Obstet Gynecol 2017.

institutions when applying for laparoscopic privileges. ${ }^{5}$ Similarly, the Laparoscopic Skills Training and Testing (LASTT) method measures 3 basic laparoscopic principles per the European

\section{FIGURE 2}

\section{Instructional video tutorials}

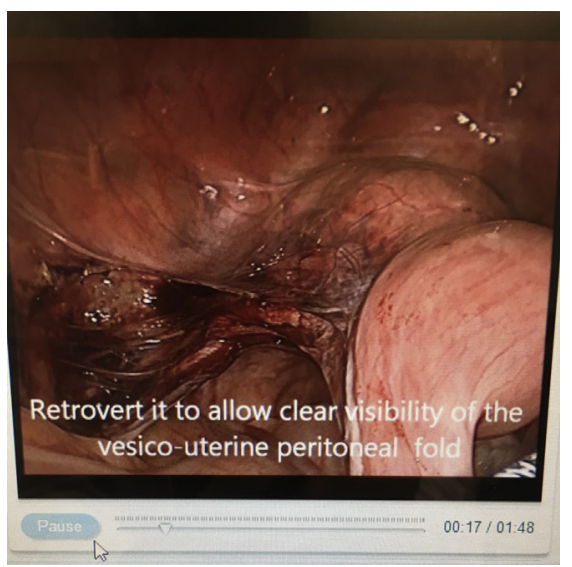

LapMentor (3D Systems Healthcare, Littleton, $\mathrm{CO}$ ) virtual-reality trainer displaying video tutorial of laparoscopic hysterectomy.

Wohlrab. Simulation and gynecologic surgical training. Am J Obstet Gynecol 2017.
Society for Gynecological Endoscopy: laparoscopic camera navigation, handeye coordination, and bimanual coordination. ${ }^{6}$ Both systems provide objective methods for evaluation of basic laparoscopic skills, but the LASTT method is geared toward laparoscopic skills unique to operating in the female pelvis.

\section{Repetition}

Along with skill generalization, repetition is a key component to simulation and paramount to skill mastery. Larsen et $\mathrm{al}^{7}$ randomized residents to laparoscopic simulation or standard clinical education prior to their first laparoscopic surgery. Blinded examiners graded the residents who had participated in simulation training as being more skilled than those who did not receive simulation. They found that the obstetrics-gynecology residents in the simulation arm performed their first laparoscopic salpingectomy at a level equivalent to residents who had completed $20-50$ prior cases. In contrast, the residents not in the simulation arm were consistently graded similar to those who had completed $<5$ cases. $^{7}$ Connor et $\mathrm{al}^{7}$ also demonstrated that repetition is the key to skill mastery. Obstetrics and gynecology residents achieved a plateau in time to completion of exercises on a laparoscopic simulator after only 10 repetitions. The plateau remained despite long gaps between sessions, suggesting that proficiency is achieved through repetition regardless of time interval between surgical simulation training. ${ }^{8}$ Defining proficiency is an area of intense focus and research, but when surgical proficiency is achieved, efficiency and surgical confidence improve. Such traits are associated with fewer intraoperative errors. 9 Furthermore, trainees may push their limits during simulation exercises. While mistakes are not welcome in the operating room, they are opportunities for further learning in the simulation laboratory. Learners can adjust their skills to avoid preventable errors, and then practice repetitively until mastery is achieved. They can also learn to troubleshoot equipment problems in a safe environment. Therefore, deliberate practice is of utmost importance in simulation training. Deliberate practice involves "engaging learners in focused, effortful skill repetition in progressive exercises that provide informative feedback." ${ }^{10}$ However, a shortcoming of simulation is the inability to recreate a world in which every injury or miscalculation is reproduced. We often encourage learners to practice with experts who can "throw a curve ball" or create an unexpected event they may encounter in the operating room.

\section{Development of gynecologic simulation models}

Medical simulation and, in particular, gynecologic surgical simulation has evolved rapidly in the past decade. Advances in technology and the approach to simulation have broadened applications to training scenarios that were not previously possible.

Early attempts at surgical simulation were hindered by compromises brought about by an immature technology. Early simulation equipment was unable to replicate surgical tools, procedures, techniques, or even the surgical environment. As laparoscopic surgery became more common, the use of low-fidelity box trainers gained popularity (Figure 1). Box trainers are useful tools to develop and refine laparoscopic skills using actual operating room equipment. Open-box trainers allow for direct observation and manipulation of instruments in the field. Once the box lid is closed, the surgeon removes him or herself from the surgical field, much like laparoscopy. The operative field becomes connected to the surgeon only by tools and tactile feedback. This can be a difficult task for new surgeons to master. Simulation exercises can be made even more difficult by attaching a laparoscope and monitor. Then, a practicing surgeon can simulate difficult visualspatial relationships, maintaining surgical orientation and object manipulation.

Advances in technology have promoted the evolution of simulation equipment. Today's high-fidelity, virtual-reality trainers include advanced computer processing that also includes haptic feedback. Many also include video simulation and tutorials of actual cases (Figure 2). They are stand-alone 
machines that take up a small footprint, and are often mobile. Although highfidelity trainers offer many software add-on options, their superiority over low-fidelity trainers is debated. Some studies compared high- and low-fidelity trainers and concluded that low-fidelity trainers are as good as their more expensive counterparts in skill acquisition. ${ }^{11,12}$ When used as a self-directed learning tool, the high price tag of high-fidelity trainers may, however, be justified. Their complex software offers the advantage of integrating computerbased tutorials in the hopes of accelerating procedural learning through a virtual expert. These tutorials are important in self-directed learning when expert teachers are not available. Unfortunately, even the best software programs are currently unable to assess intraoperative decision-making and creativity. In addition, computerized simulation cannot replace expert mentoring about preoperative judgment. Such immediate feedback is crucial to surgical training. Novice surgeons perform fewer errors with fewer repetitions when instructed by an expert surgeon. ${ }^{13,14}$ Thus, the role of the surgical mentor cannot be lost and is the cornerstone of a surgical residency. When possible, expert surgeons should assist in the development and implementation of a surgical simulation curriculum.

Although technological advances have taken simulation to a new level, it remains a challenge to replicate the intricacies of surgery. To simulate an abdominal hysterectomy, a comprehensive model would need to be built consisting not only of the uterus and attached ligaments, but also the vasculature, surrounding organs, and potential anatomic spaces. The ability to repeatedly clamp and cut pedicles and to develop surgical planes would need to be incorporated. The ideal model would need to stand up to repetitive use, and not be prohibitively expensive. Animal models have proved effective in simulating robotic hysterectomies but remain prohibitively expensive for widespread use. ${ }^{15}$ Large, fully equipped operating rooms are necessary to ensure the animals are cared for and sacrificed in

\begin{tabular}{|c|c|c|}
\hline & Expert-led simulations & Self-directed simulations \\
\hline PGY 1 & $\begin{array}{l}\text { - Boot camp } \\
\text { - Resident simulation days }\end{array}$ & $\begin{array}{l}\text { - VR laparosopic training } \\
\text { - VR robotic training } \\
\text { - Laparoscopic box trainers } \\
\text { - Suturing model }\end{array}$ \\
\hline PGY 2 & $\begin{array}{l}\text { - Hysteroscopic simulation with REI fellows } \\
\text { - Resident simulation days } \\
\text { - Clay pelvis anatomy workshop }\end{array}$ & $\begin{array}{l}\text { - VR laparosopic training } \\
\text { - VR robotic training } \\
\text { - Laparoscopic box trainers } \\
\text { - Suturing model }\end{array}$ \\
\hline PGY 3 & $\begin{array}{l}\text { - Laparoscopic simulation with } \\
\text { urogynecology fellows } \\
\text { - Resident simulation days } \\
\text { - Cadaver workshop }\end{array}$ & $\begin{array}{l}\text { - VR laparosopic training } \\
\text { - VR robotic training } \\
\text { - Laparoscopic box trainers } \\
\text { - Suturing model }\end{array}$ \\
\hline PGY 4 & $\begin{array}{l}\text { - Robotic simulation with gynecology } \\
\text { oncology fellows } \\
\text { - Resident simulation days } \\
\text { - Cadaver workshop }\end{array}$ & $\begin{array}{l}\text { - VR laparosopic training } \\
\text { - VR robotic training } \\
\text { - Laparoscopic box trainers } \\
\text { - Suturing model }\end{array}$ \\
\hline
\end{tabular}

$P G Y$, postgraduate year; $R E I$, reproductive endocrinology and infertility; $V R$, virtual reality.

Wohlrab. Simulation and gynecologic surgical training. Am J Obstet Gynecol 2017.

humane ways. In addition, differences in anatomy may limit the usefulness of certain procedures. ${ }^{16}$

Synthetic cadavers avoid the ethical concerns of animal surgical laboratories. The SynDaver cadaver (SynDaver Labs, Tampa Bay, FL) is one such simulation aid that allows for surgical practice via extremely detailed and lifelike reproductions of every bone, muscle, vascular component, and organ. But at $\$ 60,000$ per simulated cadaver, the costs preclude widespread use. Many institutions have a limited simulation budget and thus need to employ less expensive options.

\section{Bringing simulation into your residency}

The development of a simulation curriculum is important to its implementation and success. A formalized simulation curriculum will enhance resident training throughout all 4 years (Table). Learning modules need to allow for focused teaching and efficient use of time. The core concept of skill repetition should be encouraged and available through both skill generalization and skill transfer exercises. Learning objectives should be clear with each simulation model. Sample learning objectives for the simulation of gynecologic surgeries published by the American Congress of Obstetricians and Gynecologists Simulations Working Group are available for: (1) vaginal hysterectomy, (2) laparoscopic salpingectomy, (3) laparoscopic salpingo-oophorectomy, and (4) laparoscopic ovarian cystectomy. ${ }^{17}$ Even though simulation has been integral to the training of obstetrics and gynecology residents in many academic institutions, there is no standardized curriculum across residencies.

Designing a structured curriculum for gynecologic simulation is a daunting task. As a basis for future learning, postgraduate year- 1 is for orientation to surgical tools and simulation equipment. At our institution, this is accomplished during "boot camp week," ie, the first week of residency. Incoming residents spend 4-6 hours with attending physicians and operating room staff reviewing types of sutures, retractors, and basic surgical instruments. It is expected that they have core knowledge of the supplies they will be using before they enter the operating room. This high-yield exercise also helps to conquer regional variations on names for the same surgical instrument. During this time frame, we also orient our trainees to 


\section{FIGURE 3}

\section{Virtual-reality teaching aids}

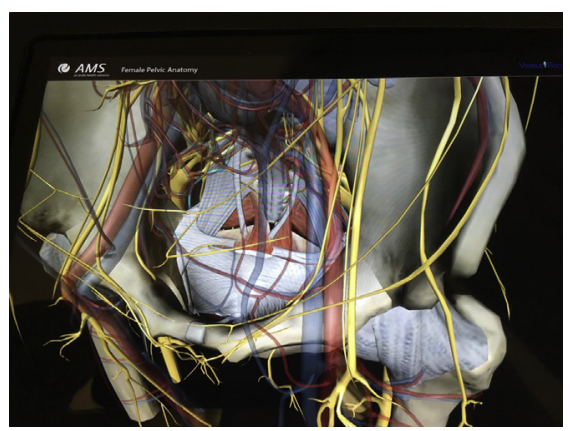

Software allows for rotation, labeling, and manipulation of pelvic structures including nerves and vessels (AMS Visible Body, Boston, MA).

Wohlrab. Simulation and gynecologic surgical training. Am J Obstet Gynecol 2017.

the location and use of the simulation equipment. Providing unlimited access encourages learners to use simulation equipment often. Ideally, surgical simulation equipment should be near the operating room; this allows residents to practice before, between, and after actual surgical cases.

Next is a progression to basic procedural techniques and anatomical education. Brydges et $\mathrm{al}^{18}$ demonstrated that learners prefer a progressive curriculum from low- to high-fidelity with facilitated self-directed learning. Computerbased teaching models and low-fidelity silicone replicas demonstrate the location of anatomic structures and their relationships in a 3-dimensional space ${ }^{19}$ (Figure 3). Our residents participate in a clay pelvis workshop to further illustrate pelvic anatomy. ${ }^{20}$ Having upper-level residents review these concepts with their junior residents encourages mastery at both levels. Once anatomic knowledge is mastered, the learner can progress onto more complex and procedure-specific techniques (eg, clamping, cutting, and knot-tying).

Learning objectives are tailored to the level of proficiency within each training program. During resident simulation days (3 times per year), residents are taught important surgical techniques by faculty. For vaginal surgery, we employ low-fidelity models that require trainees to place clamps and suture within a plastic cup. To teach vaginal morcellation, we have used chicken breasts extracted through the opening of a milk container. ${ }^{21}$ In laparoscopic, hysteroscopic, and cystoscopic surgery, learners are instructed how to manipulate angled scopes to achieve desired views above and around anatomy. Residents practicing laparoscopic and robotic surgery progress to object manipulation. We encourage learners to experiment with hand dominance and encourage ambidexterity, as well as selfdirected practice on simulators during downtime. Experimentation is often encouraged in simulation; trainees may push their limits during simulation exercises without fear of harm to patients or criticism of their own skills. We refer to our simulation areas as judgement-free zones. This promotes use of the equipment across levels and aptitudes toward a common goal: improvement.

As the learner becomes more adept with basic laparoscopic skills, the tasks are advanced. Many high-fidelity virtualreality simulators are prohibitively expensive during the initial investments into surgical simulation, but low-fidelity equipment is a viable option. Laparoscopic box trainers may be purchased or even homemade. Two-handed object manipulation in laparoscopy is easily taught with a peg transfer exercise. Progressing to pattern cutting and eventually to intracorporeal knot-tying is expected by the third year of residency. The complexity of surgery is often related to its proximity to vital structures. Although our junior residents learn and practice extracorporeal laparoscopic suturing, they are not expected to be able to place retropubic sutures adjacent to the obturator neurovascular bundle during a paravaginal repair; that is typically reserved for chief residents or fellows. But this early simulation will better prepare them for complex procedures when they are senior residents. ${ }^{22,23}$ Currently, we do not grade or evaluate residents during simulation exercises.

Our simulation curriculum also includes dedicated sessions with expert-led modules. These sessions augment surgical learning in a protected group session. Third- and fourth-year residents participate in cadaver workshops that focus on bowel and urologic injuries. They are taught recognition and repair techniques by gynecologic oncology, urogynecology, colorectal, and urology staff. Local experts are an excellent resource to teach procedural techniques, tips, and tricks. They are also helpful in sharing medical decision-making, anecdotes, and surgical improvisation. Highvolume surgeons have improved outcomes and fewer complications, and their insight during training sessions helps accelerate learning outside the expensive confines of an operating room. ${ }^{24-26}$ We have used these expert-led sessions not only for trainees, but also for lower-volume surgeons, for those returning from an extended leave of absence, or during the early learning phase of a new procedure or technique.

Designing a novel simulation curriculum is often limited by time, money, and space. High-fidelity laparoscopic trainers are often expensive costing $>\$ 100,000$. In addition, the warranties for such systems approach $\$ 15,000$ annually. Cost-sharing models with other departments (eg, general surgery) will defray costs, but will also increase utilization along with wear and tear. Justifying the cost of simulation equipment requires a careful look at its return on investment. The return on investment for simulation is typically measured in operating room efficiency and decreased surgical errors. Surgical simulation has been shown to increase surgical confidence, which is linked with fewer surgical errors. ${ }^{10}$ A recent Cochrane review concluded that virtualreality surgical simulation was associated with decreased total operating room time, with an increase in amount of surgery performed by residents. Whether these surrogate markers translate into improved surgical education and excellence in clinical care has yet to be proven. ${ }^{27}$ Many commercial simulation systems are trying to capture such data through integrative web-based networks between institutions. These systems allow centers to share and 
customize learning modules. Through these collaborations, a new breed of research has been born: one that examines the utility, effectiveness, and validity of surgical simulators.

The validation of surgical simulation curricula remains an area of exploration. There are only a few standardized measures to assess an individual's level or progress during simulation. The Objective Structured Assessment of Technical (OSAT) skills process is a commonly used method of assessing procedural competency. The process involves a series of faculty-staffed stations where learners rotate and perform a series of specific psychomotor tasks relevant to their specialty. Learners are assessed using global and checklist assessments. The OSAT process is also used to evaluate decisionmaking while performing a specific procedure. While applicable to a specific set of tasks, OSAT is difficult to apply to the performance of a complete surgical procedure such as a laparoscopic hysterectomy. In this case, the key steps are usually teased out and the learner is instructed to perform only those key steps during the assessment. As discussed previously, the FLS and LASTT methods can also be used to objectively describe laparoscopic skills. But as a previous Cochrane review noted, there are currently no studies of surgical simulation demonstrating an effect on morbidity, mortality, quality of life of patients, or hospital stay. ${ }^{27}$ Therefore, we recommend using these validated assessment tools to describe the progress, plateau, and learning curves of simulation training and the relation of simulation skills to actual operative performance.

In summary, a gynecologic surgical simulation curriculum should be developed to enhance and supplement current obstetrics and gynecology residency training. It should emphasize skill repetition through both skill generalization and skill transfer, utilizing both low-fidelity and high-fidelity trainers. A successful simulation curriculum should be focused on 3 important considerations: (1) unrestricted access to surgical simulation equipment; (2) opportunity to use a combination of self-directed and facilitated learning by qualified supervising surgeons; and (3) systematic training sessions with the aid of expert surgeons to provide immediate feedback and instruction. Simulation training allows learners to make and learn from their mistakes, repeat procedures multiple times to improve muscle memory, and enhance skill competency with the aid of formative feedback. $^{28}$ As medical advancements continue to transform the surgical arena, simulation will play an even larger role in training our future residents.

\section{REFERENCES}

1. Magrina JF. Isn't it time to separate the $O$ from the G? J Minim Invasive Gynecol 2014;21: 501-3.

2. Guntupalli SR, Doo DW, Guy M, et al. Preparedness of obstetrics and gynecology residents for fellowship training. Obstet Gynecol 2015;126:559-68.

3. Rutkow R. The education, training, and specialization of surgeons. Turn-of-the-century America and its postgraduate medical schools. Ann Surg 2013;258:1130-6.

4. Gallagher AG, Ritter EM, Champion H. Proficiency-based training as a paradigm shift in surgical skills training. Ann Surg 2005;241:364-72. 5. Okrainec A, Soper NJ, Swanstrom LL, Fried GM. Trends and results of the first 5 years of fundamentals of laparoscopic surgery (FLS) certification testing. Surg Endosc 2011;25:1192-8.

6. Rudi, et al. A valid model for testing and training laparoscopic psychomotor skills. Gynecol Surg 2010;7:133-41.

7. Larsen CR, Soerensen JL, Grantcharov TP, et al. Effect of virtual reality training on laparoscopic surgery: randomized controlled trial. BMJ 2009;338:b2074.

8. Connor EV, Raker C, Wohlrab KJ. Effects of repetition and inactivity on laparoscopic skills training. J Minim Invasive Gynecol 2016;23:194-7. 9. Seymour NE, Gallagher AG, Roman SA, et al. Virtual reality training improves operating room performance. Results of a randomized, doubleblinded study. Ann Surg 2002;236:458-64.

10. Thomas GW, Johns BD, Marsh JL, Anderson DD. A review of the role of simulation in developing and assessing orthopedic surgical skills. lowa Orthop J 2014;34:181-9.

11. Diesen DL, Erhunmwunsee L, Bennett KM, et al. Effectiveness of laparoscopic computer simulator versus usage of box trainer for endoscopic surgery training for novices. J Surg Educ 2011;68:282-9.

12. Norman G, Dore K, Grierson L. The minimal relationship between simulation fidelity and transfer of learning. Med Educ 2012;46:636-47. 13. Van Sickle KR, Gallagher AG, Smith $C D$. The effect of escalating feedback on the acquisition of psychomotor skills for laparoscopy. Surg Endosc 2007;21:220-4.

14. Trehan A, Bartnett-Vanes $A$, Carty $M J$, McCulloch P, Maruthappu M. The impact of feedback of intraoperative technical performance in surgery: a systematic review. BMJ Open 2015;5:e006759.

15. Hoffman MS. Simulation of robotic hysterectomy utilizing the porcine model. Am J Obstet Gynecol 2012;206:523e1-2.

16. Hoffman MS, Ondrovic LE, Wenham RM, et al. Evaluation of the porcine model to teach various ancillary procedures to gynecologic oncology fellows. Am J Obstet Gynecol 2009;201:116.e1-3.

17. ACOG. Ob-Gyn simulations curricula. Available at: http://www.acog.org/AboutACOG/ACOG-Departments/Simulations-Cons ortium/OB-GYN-Simulations-Curricula.

18. Brydges R, Carnahan $H$, Rose $D$, Dubrowski A. Comparing self-guided learning and educator-guided learning formats for simulation-based clinical training. J Adv Nurs 2010;66:1832-44.

19. Barrier BF, Thompson $A B$, McCullough MW, Occhino JA. A novel and inexpensive vaginal hysterectomy simulator. Simul Healthc 2012;7:374-9.

20. Myers DL, Arya LA, Verma A, Polseno DL, Buchanan EM. Pelvic anatomy for obstetrics and gynecology residents: an experimental study using clay models. Obstet Gynecol 2001;97:321-4.

21. Shaw J, Wohlrab K. Transvaginal morcellation simulation model. J Minim Invasive Gynecol 2015;22:s120.

22. Rackow BW, Solnik MJ, Senapati S, Pozolo KE, Du H. Deliberate practice improves obstetrics and gynecology residents' hysteroscopy skills. J Grad Med Educ 2012;4:329-34.

23. Fann Jl, Calhoon JH, Carpenter AJ, et al. Simulation in coronary artery anastomosis early in cardiothoracic residency training: the boot camp experience. J Thorac Cardiovasc Surg 2010;139:1275-81.

24. Flood AB, Scott WR, Ewy W. Does practice make perfect? Part II: the relation between volume and outcomes and other hospital characteristics. Med Care 1984;22:115-25.

25. Vree FE, Cohen SL, Chavan N, Einarsson J. The impact of surgeon volume on perioperative outcomes in hysterectomy. JSLS 2014;18: 174-81.

26. Munro M. Surgical simulation: where have we come from? Where are we now? Where are we going? J Minim Invasive Gynecol 2012;19:272-83. 27. Nagendran M, Gurusamy KS, Aggarwal R, Loizidou M, Davidson BR. Virtual reality training for surgical trainees in laparoscopic surgery. Cochrane Database Syst Rev 2013;8: CD006575.

28. Goff BA. Changing the paradigm in surgical education. Obstet Gynecol 2008;112:328-32. 